\title{
Ejercicio integrador para el estudio de microprocesadores del ingeniero en telecomunicaciones y electrónica
}

\author{
David Castro-Piñol ${ }^{a}$ Bernardino Enrique Álvarez-Portuondo ${ }^{b}$ Humberto Hervella-Mateos ${ }^{c}$ \\ \& Ilen Rivero-Pouymiró ${ }^{d}$ \\ ${ }^{a}$ Centro de Estudios de Neurociencias, Procesamiento de Imágenes y Señales (CENPIS), Universidad de Oriente, Santiago de Cuba, Cuba. \\ davidpinol@uo.edu.cu \\ ${ }^{b}$ Departamento de Ingeniería Biomédica, Universidad de Oriente, Santiago de Cuba, Cuba. balvarez@uo.edu.cu \\ ${ }^{c}$ División Territorial Santiago de Cuba (DTSC), ETECSA, Cuba, humberto.hervella@etecsa.cu \\ ${ }^{d}$ Departamento de Ingeniería en Telecomunicaciones, Universidad de Oriente, Santiago de Cuba, Cuba. ilen@uo.edu.cu
}

\begin{abstract}
Resumen - En la enseñanza de la ingeniería en los últimos años resulta difícil lograr la integración de conocimientos y habilidades para alcanzar un aprendizaje holístico y efectivo en el diseño de sistemas complejos. Este trabajo tiene como objetivo realizar una propuesta de ejercicio académico integrador para la asignatura de Microprocesadores de la carrera de Telecomunicaciones y Electrónica en el contexto de la Universidad de Oriente para fomentar la formación integral del ingeniero. La propuesta consta del diseño de un modem con el microcontrolador 8051 y la simulación de una transmisión de caracteres. Esta propuesta contiene una explícita integración horizontal y vertical con habilidades y conocimientos de las disciplinas: Sistemas de Telecomunicaciones, Teoría de la Información y Sistemas Electrónicos que son parte esencial de la formación del profesional en ingeniería en Telecomunicaciones y Electrónica. Para el diseño se propone el uso de la plataforma MATLAB e ISIS Proteus.
\end{abstract}

Palabras Clave- interdisciplinariedad, enseñanza de las Telecomunicaciones y Electrónica, módem, microcontrolador 8051.

Recibido: 1 de febrero de 2017. Revisado: 25 de mayo de 2017. Aceptado: 2 de junio de 2017

\section{Microprocessor integrator exercise for the telecommunications and electronic engineer}

\begin{abstract}
In engineering teaching is important the skills and knowledge integration in order to achieve a holistic and effective learning in complex systems design. In this paper, it is proposed an academic integrator exercise for the Microprocessor subject of the Telecommunications and Electronic career in order to advance the engineering integral education at Universidad de Oriente. This exercise is a modem design based on the 8051 microcontroller. Moreover, MATLAB and ISIS Proteus platform was used in order to simulate a transmission in ASK modulation. This proposal contain an explicit horizontal and vertical integration with skills and knowledges of: Telecommunications Systems, Information Theory and Electronic Systems which are a professional formation essential part of Telecommunications and Electronic engineer.
\end{abstract}

Keywords - interdisciplinary, Telecommunications and Electronic teaching, modem, 8051 microcontroller.

\section{Introducción}

En las últimas décadas, la humanidad ha experimentado cambios sin precedentes en diversos ámbitos del mundo social y tecnológico, generando inmensos desafíos tanto a las personas, como a las organizaciones, quienes deben desenvolverse en escenarios más complejos, dinámicos e inciertos. Diferentes estudios sobre el rol de la educación la definen como el instrumento fundamental para preparar las próximas generaciones. Jacques Delors, en la presentación del Informe a la UNESCO, de la Comisión Internacional sobre la educación para el siglo XXI [1], lo afirma cuando plantea que: "frente a los numerosos desafíos del porvenir, la educación constituye un instrumento indispensable para que la humanidad pueda progresar hacia los ideales de paz, libertad y justicia social". De manera que los esfuerzos humanos por mejorar y crear metodologías en contextos complejos son necesarios para el avance de la educación. Una de las áreas que presenta mayor desafíos en el contexto actual es la enseñanza de la ingeniería.

La formación del ingeniero hoy en día presenta alta complejidad debido a la gran cantidad y diversa información disponible, problemas a los que enfrenta y a la globalización de los mercados. Las estructuras corporativas son cada vez más participativas, exigiéndole al profesional mayor trabajo en equipo y responsabilidad en la toma de decisiones. La enseñanza de la ingeniería en nuestro contexto presenta algunas limitaciones porque existen tendencias a la transmisión de los fundamentos teóricos propios de cada una de las profesiones y prevalece la metodología tradicional, donde prima la enseñanza descontextualizada, el aprendizaje memorístico y la convencional división de los saberes entre disciplinas.

Se hace entonces necesario, en pro del desarrollo, reconocer que en la sociedad actual se presenta, como nunca antes, la movilidad del conocimiento, lo que trae como consecuencia la imperativa actualización de las profesiones, disciplinas y la tendencia al trabajo interdisciplinar como modelo posibilitador de producción de conocimiento.

La ingeniería es una profesión que se basa en las disciplinas, es decir, es interdisciplinaria por naturaleza. La interdisciplinariedad es el método en que la cooperación entre

Como citar este artículo: Castro-Piñol, D., Álvarez-Portuondo, B.E., Hervella-Mateos, H. and Rivero-Pouymiró, I., Ejercicio integrador para el estudio de microprocesadores del ingeniero en telecomunicaciones y electrónica. Educación en Ingeniería, 12(24), pp. 43-51, Julio, 2017. 
varias disciplinas provoca intercambios reales, existiendo reciprocidad. Se evidencia cuando se plantea un problema en términos tales que no puede ser resuelto desde una sola disciplina. En este sentido, en la enseñanza de la ingeniería la interdisciplinariedad proporciona una gran riqueza en cuanto a las posibilidades que le brinda al estudiante para el aprendizaje significativo, ya que le permite abordar los contenidos científicos desde varios puntos de vista y adquirir una concepción más amplia, dándole un sentido mucho más completo al relacionarlo con otras áreas del conocimiento [2] [3]. Una metodología que se encuentra directamente relacionada con la interdisciplinariedad y que busca acercar al estudiante a la solución de problemas del mundo real es el Aprendizaje Basado en Problemas, ABP [4].

Barrows 1986 [4] define al ABP como "un método de aprendizaje basado en el principio de usar problemas como punto de partida para la adquisición e integración de los nuevos conocimientos”. En el ABP se parte de una situación problemática, donde la experiencia se debe centrar en el análisis, diseño y experimentación de una solución donde los protagonistas del aprendizaje son los propios alumnos. Esta metodología se encuentra muy relacionada con la interdisciplinariedad ya que los alumnos necesitan recurrir a conocimientos adquiridos en diversas asignaturas para intentar solucionar un problema [5]. Existen algunos trabajos que muestran los resultados positivos de la aplicación del ABP en problemas de ingeniería [6,7].

La carrera de ingeniería en Telecomunicaciones y Electrónica en la Universidad de Oriente es de perfil amplio por la cantidad y variedad de disciplinas que agrupa. Se destaca el carácter interdisciplinario que deben lograr sus asignaturas. Sin embargo existen asignaturas en las que la integración de conocimientos resulta escasa. Se cree que este fenómeno se debe en mayor parte, a la complejidad de los contenidos y la poca existencia de proyectos de carácter integrador que requieran del trabajo en equipos, principalmente ejercicios que se acerquen más al perfil profesional del ingeniero en Telecomunicaciones y Electrónica.

Como posible alternativa a la escasez de tareas de carácter integrador, el presente trabajo tiene como objetivo la propuesta y resolución técnica y metodológica de un ejercicio de carácter integrador del perfil profesional del ingeniero en Telecomunicaciones y Electrónica. También se pretende mostrar la solución de este ejercicio con la integración de las competencias disciplinarias de la carrera. El ejercicio consta del diseño y simulación de un módem con el microcontrolador 8051 que es el más visto en la carrera y el estudio de su modelo es básico para la comprensión de otros microcontroladores. La propuesta puede ser considerada como tarea final extra clase de la asignatura Microprocesadores II del primer semestre de cuarto año de la carrera ingeniería en Telecomunicaciones y Electrónica. Aunque este trabajo no analiza un enfoque completo de ABP sobre el ejercicio, sí es aconsejable orientar su resolución mediante su metodología debido al carácter holístico del ejercicio y que está concebido para el trabajo en equipo. La propuesta pretende formar en los estudiantes capacidades competentes en la solución de problemas interdisciplinarios de la profesión.

El ejercicio propuesto tiene un carácter interdisciplinario porque fue diseñado para que fuese resuelto desde la colaboración y relación entre diferentes disciplinas y no desde un enfoque aislado. Debido a esto la propuesta motiva al trabajo en equipo en los estudiantes para darle solución. En un equipo de trabajo de estudiantes de ingeniería, generalmente existe una tendencia a que cada uno tenga preferencia por determinada disciplina o le sea más fácil desarrollar algunas habilidades específicas dentro de su profesión. De ahí que se plantea que el trabajo en equipo con este ejercicio puede fortalecer interdisciplinariedad, la colaboración entre sus miembros, la comprensión y el respeto.

Cabe destacar el grado integrador del ejercicio a nivel horizontal y vertical con las principales disciplinas de la carrera. En la Fig. 1 se puede apreciar un diagrama de relaciones donde se aprecia la relación del ejercicio con asignaturas de la carrera.

Se puede observar en la Fig. 1 la relación del ejercicio integrador propuesto con el nivel básico, con el semestre, con el año y con el nivel profesional. El nivel básico agrupa todas las asignaturas básicas que son necesarias para resolver la propuesta. En el semestre son las asignaturas que se encuentra recibiendo el estudiante a las cuales aporta y se nutre la propuesta. En el año es la asignatura del próximo semestre que se relaciona con la propuesta. En el nivel profesional que es el quinto año, es la asignatura a la que el ejercicio aporta aunque en menor medida. Estas relaciones pretenden mostrar las competencias que los estudiantes han desarrollado en cada asignatura que son necesarias para el diseño del ejercicio y cómo todo este conjunto de conocimientos, habilidades, aptitudes y actitudes se integran y refuerzan en esta experiencia particular. En las siguientes secciones a medida que se resuelve cada etapa del diseño se muestra cómo se evidencia la interdisciplinariedad entre las asignaturas expuestas.

Este documento está compuesto por cuatro secciones principales. La primera sección es la introducción. La segunda sección cubre la metodología y las técnicas utilizadas para la resolución del ejercicio donde se abordan las diferentes etapas del diseño y sus relaciones con las disciplinas involucradas. La tercera sección analiza los resultados obtenidos que prueban el correcto funcionamiento del diseño y una cuarta sección que comprende las conclusiones del trabajo y posibles áreas para trabajar en el futuro.

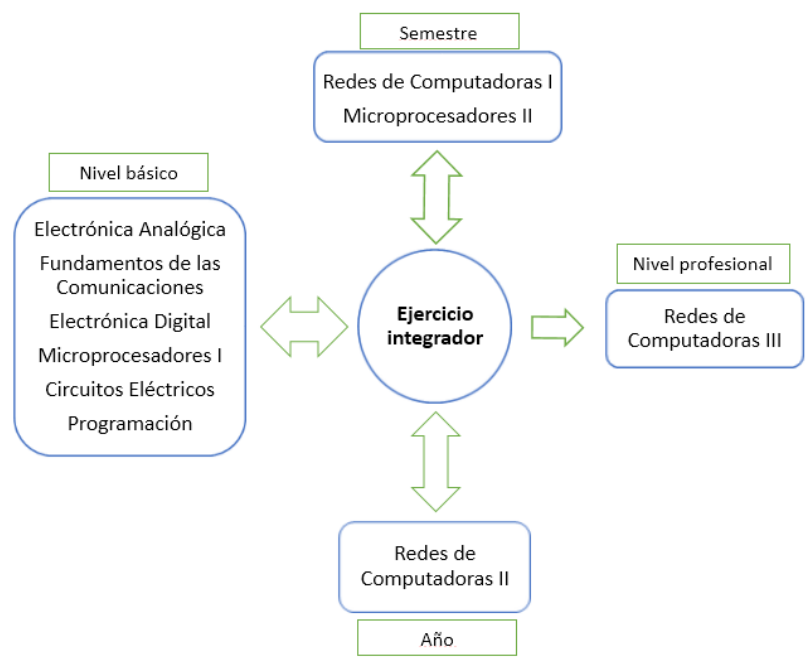

Figura 1. Diagrama de relaciones del ejercicio integrador con las asignaturas. Fuente: Los autores 
Un conjunto de materiales anexos se encuentra en: https://www.dropbox.com/sh/8f5gcyfuck1dtoh/AADQ_4i0bH YTzyewr5i1uJb1a?dl=0 para ser utilizados con fines investigativos y docentes.

\section{Metodología para resolver el ejercicio}

La elaboración y orientación del ejercicio es una parte fundamental de la tarea a presentar a los estudiantes. Debe ser precisa, lo suficientemente compleja (pero no imposible) y nueva para despertar la motivación de los estudiantes en la experimentación y búsqueda de una solución. Por las características de este ejercicio es importante darle libertad al estudiante a la hora de escoger un camino para darle solución. A continuación se plantea el ejercicio a resolver como posible ejemplo de tarea final extra clase.

Ejercicio: Diseñar un módem con el microcontrolador 8051 con sus operaciones básicas que sea capaz de transmitir información usando modulación Amplitude-Shift Keying (ASK) [8] sobre el canal telefónico. Realizar una simulación en Proteus ISIS donde se compruebe la modulación y desmodulación de una cadena de caracteres entre transmisor y receptor. El sistema debe contener un protocolo simple de comunicación que sea capaz de detectar errores en la transmisión.

La modulación constituye uno de los conceptos más importantes de la carrera por eso la significancia del diseño de un módem. Los módems en general constituyen el equipamiento encargado de realizar las labores de modulación y desmodulación de las señales que se intercambian a través de la red telefónica y entre dos equipos terminal de datos (ETD). El módem tiene como función la modulación de una señal digital, convirtiéndola en una señal analógica que se transmite a través de un medio y demodula dicha señal para recuperar la información original. Hacer énfasis de que se diseña un módem porque se tiene en cuenta más las operaciones de modulación y desmodulación que otras funciones que traen los módems.

También se aprecia la orientación del ejercicio con el microcontrolador 8051 debido a que es el microcontrolador que los estudiantes más han estudiado en la carrera donde ya tienen consolidados conocimientos y habilidades sobre el mismo. No se propone este microcontrolador porque sea el mejor para el diseño de un módem.

\subsection{Simulación en Simulink de la modulación y desmodulación}

Después del planteamiento del ejercicio, se motiva a los estudiantes a que realicen entre ellos una lluvia de ideas en busca de organizar, planificar y distribuir la resolución del problema de diseño. El profesor debe interactuar como mediador y facilitador del diálogo con los estudiantes en esta etapa de análisis ya que contribuye a prepararlos en el ejercicio del trabajo en equipo y cómo deben articular entre ellos sus conocimientos y habilidades.

Es conveniente orientar el comienzo realizando un diseño que vaya desde lo más general hasta lo más particular. Es aconsejable también comenzar realizando cálculos generales del sistema, simulaciones y programar operaciones básicas en el microcontrolador 8051 para posteriormente diseñar el esquema eléctrico general.

Una forma para comenzar es confeccionar la estructura del diseño en un esquema general en bloques para determinar parámetros y características de la comunicación. Una herramienta muy útil para comprobar y analizar el diseño general es el Simulink que está integrado a MATLAB. El estudiante en este punto de la carrera ha desarrollado las habilidades básicas en el uso del Simulink de años anteriores especialmente en asignaturas de la disciplina Teoría de la Información.

Simulink es un entorno de diagramas de bloque para la simulación multidominio y el diseño basado en modelos. Admite el diseño y la simulación a nivel de sistema, la generación automática de código y la prueba y verificación continuas de los sistemas embebidos.En la Fig. 2 se aprecia el esquema propuesto en Simulink de la transmisión y recepción de información por el canal telefónico usando la modulación ASK. Se observan los bloques: generador aleatorio de señal digital (Random Integer Generator), portadora local (Sine Wave), un modulador balanceado (Product), el canal telefónico, el modulador balanceado de la recepción (Product 1) con una portadora sincronizada (Sine Wave 1), un filtro paso bajo, un comparador de umbral (Compare To Constant) y el osciloscopio (Scope).

El estudiante puede llegar al esquema de la Fig. 2 sin mucho esfuerzo porque en el semestre en que se encuentra ha recibido el tema de modulaciones digitales correspondiente a la asignatura de Fundamentos de las Comunicaciones III y ha realizado simulaciones en Simulink con diferentes tipos de modulaciones digitales incluyendo ASK que es uno de los casos más simples. Este es el primer momento en el que el estudiante comienza a aplicar contenidos y habilidades horizontales de otras asignaturas que se encuentra recibiendo.

Como se trata de un modem que va a transmitir por el canal telefónico hay que tener en cuenta que el rango de frecuencias del canal va de $300 \mathrm{~Hz}-3400 \mathrm{~Hz}$. En la Fig. 2 se simula como un filtro pasa banda de color rojo. La etapa que se encuentra antes del canal corresponde al modulador balanceado de ASK. Como modular es trasladar a la frecuencia de la portadora $(f c)$, el espectro del mensaje para que pueda transmitirse por el canal, entonces el valor ideal de la frecuencia de la portadora es 1850 $\mathrm{Hz}$ ya que se encuentra centrada entre $300 \mathrm{~Hz}$ y $3400 \mathrm{~Hz}$.

Después de tener el valor de la frecuencia de la portadora, el estudiante debe proceder a calcular la velocidad de transmisión $\left(V_{t}\right)$. La velocidad de transmisión máxima teórica o capacidad del canal dado el ancho de banda del canal se calcula por la fórmula de Nyquist [9] en condiciones sin ruido:

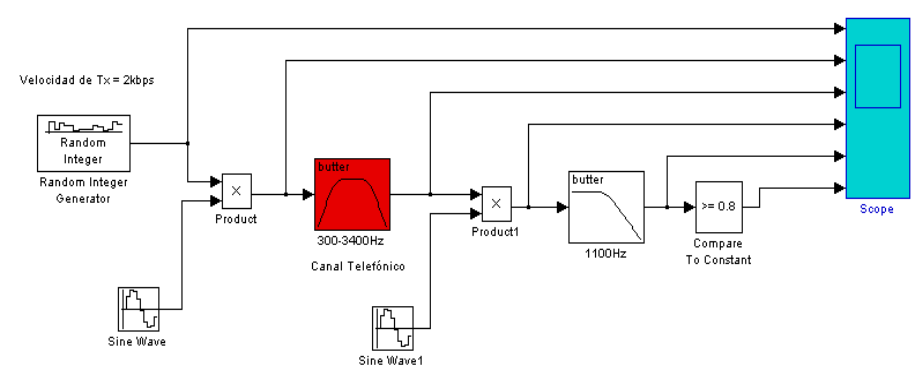

Figura 2. Esquema en Simulink de la modulación y desmodulación ASK Fuente: Los autores 


$$
C=2 W \log _{2}(n)
$$

Donde $C$ es la capacidad del canal y se mide en bits por segundo (bps). $W$ es el ancho de banda del canal que se presenta en $\mathrm{Hz}$ y $n$ es el número de niveles diferentes que puede adoptar la señal. En este punto el estudiante conoce que $W=1550 \mathrm{~Hz}$ y puede tomar $n=2$. Entonces la capacidad del canal máxima teórica es $C=3,1 \mathrm{kbps}$.

En esta simulación se ha utilizado como $V_{t}=2 \mathrm{kbps}$ y la $f_{c}=$ $2 \mathrm{kHz}$ porque son valores convenientes a los que probablemente el estudiante llegará cuando comience a enfrentarse a diversos problemas. Estos problemas están relacionados con el hecho de que valores obtenidos en cálculos teóricos no son los más adecuados a la hora de implementar en el hardware el diseño, específicamente en el microcontrolador 8051 que no tiene muy altas prestaciones. Esta secuencia de problemas y la relación entre lo teórico y práctico es muy ventajoso para cualquier ejercicio que se pretenda resolver con una metodología que integre conocimientos y habilidades de diversas disciplinas.

En la Fig. 3 se puede apreciar los resultados del osciloscopio (Scope) donde se observa la señal en las diferentes etapas de la transmisión y recepción. Esta simulación es la base para implementar posteriormente el hardware y el software que realice las operaciones observadas en cada etapa del esquema de Simulink.

Se resume cómo esta primera parte de diseño y simulación general de este módem influye en el desarrollo de competencias en los estudiantes. Primero se destaca los beneficios de la sinergia inicial en el equipo guiado por el profesor que son el enriquecimiento de las capacidades comunicativas y de interacción social frente a un conflicto técnico. Esto se debe a que en el ejercicio no se les hace explícito qué herramientas utilizar para un diseño general. Segundo, se desarrolla un pensamiento heurístico en el estudiante al darse cuenta de la posibilidad de utilizar el Simulink como primer paso para comprobar el funcionamiento del módem desde una perspectiva teórica y matemática. Como último se fortalecen las habilidades y destrezas técnicas en el uso del Simulink al construir un esquema general donde el estudiante debe configurar los parámetros de funcionamiento.

\subsection{Implementación del transmisor}

En esta sección se trata sobre la implementación del transmisor en el microntrolador 8051 donde su principal función será la modulación de una señal de datos en ASK. En la medida que se avance en el diseño técnico se va explicando la integración de las disciplinas que necesita el estudiante para esta etapa.

En la generación de la señal modulada en ASK, la señal de datos binaria entra a un modulador balanceado con la portadora como se pudo apreciar en la Fig. 2. Si se observan los resultados del osciloscopio de las dos primeras señales en la Fig. 3 se puede traducir a que cuando haya un ' 1 ' en la señal de datos binaria se transmite la portadora y cuando haya un ' 0 ' se detiene la transmisión de la portadora. ASK consiste en la conmutación de la portadora en el tiempo que dure un bit. El valor $2 \mathrm{kHz}$ de la frecuencia de la portadora es conveniente porque permite transmitir al menos un período de la misma en cada bit teniendo en cuenta que la velocidad de transmisión es $2 \mathrm{kbps}$.

\subsubsection{Sinusoide portadora}

Al conocer la simplicidad de cómo generar la señal ASK entonces el estudiante debe proceder a implementar esta etapa en el microcontrolador 8051. El primer desafío es el de generar una sinusoide que represente la portadora. Este es un punto de discusión y análisis entre los estudiantes puesto que permite el debate de técnicas para programar la generación de la sinusoide. Como la generación de una sinusoide en un microcontrolador es una tarea que el estudiante no ha enfrentado anteriormente y existen diversas vías para realizarse esta situación motiva a una síntesis de habilidades de procesamiento digital y analógico.

Una de las formas más simples para darle solución a este problema es grabar en la memoria de programa del microcontrolador una tabla de valores constantes de un período de la sinusoide. Posteriormente extraer este conjunto de valores por un puerto del microcontrolador cada un intervalo de tiempo $T_{s}$ con la ayuda de un timer. Estos valores obtenidos son posteriormente suavizados con un filtro pasa bajo.

Para calcular $T_{S}$ hay que considerar la cantidad de muestras por período que son necesarias para lograr la frecuencia de la portadora. Las ec. (2)-(4) explican mejor la relación entre estos parámetros.

$$
\begin{gathered}
N T_{s}=T_{c} \\
f_{c}=\frac{1}{N T_{s}} \\
T_{S}=\frac{1}{N f_{c}}
\end{gathered}
$$

Donde $N$ es la cantidad de muestras por período, $T_{S}$ es el período de muestreo, $T_{c}$ es el período de la portadora y $f_{c}$ la frecuencia de la portadora. Conociendo que $f_{c}=2 \mathrm{kHz}$ y tomando el valor de $N=12$ se obtiene que Ts $=42$ us.

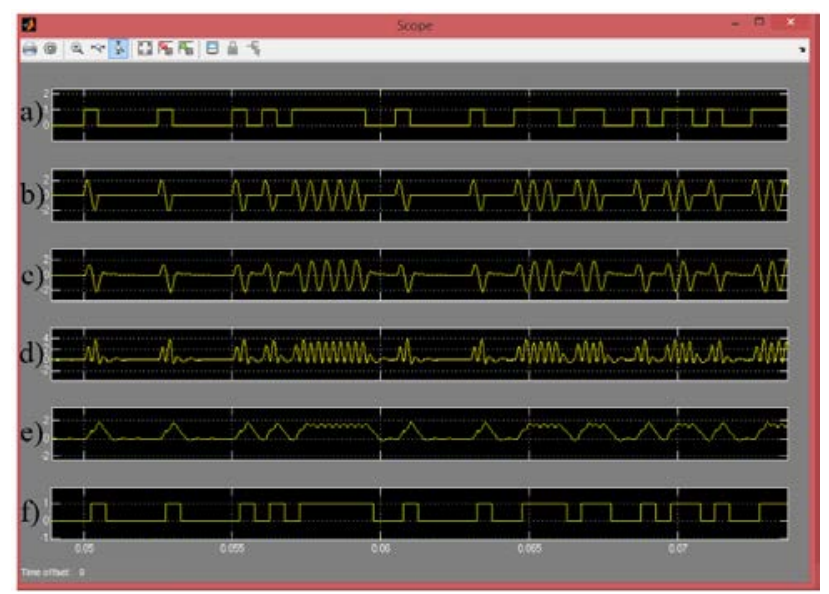

Figura 3. Etapas de la señal en el osciloscopio. a) Información a transmitir, b) información modulada en ASK, c) señal de ASK a la entrada del receptor, d) señal ASK a la salida del demodulador balanceado, e) señal filtrada, f) señal de información transmitida recuperada en el receptor.

Fuente: Los autores

Este valor de Ts corresponde a una frecuencia de muestreo de fs $=23.8 \mathrm{kHz}$ de la sinusoide portadora de $2 \mathrm{kHz}$ cumpliendo el teorema de muestreo de Nyquist. Se puede observar como en 
este diseño el estudiante debe utilizar uno de los teoremas más importantes de la carrera recibido en la asignatura de Fundamentos de Comunicaciones II a la vez que fortalece la comprensión del teorema en otro contexto

De esta forma mediante la interrupción del timer 0 que se desborda cada un tiempo $T_{S}$ se extraen los valores de la tabla para lograr formar la portadora. Los $N$ valores de la tabla se pueden obtener mediante un programa en MATLAB que ayude inicialmente al estudiante a graficar la sinusoide cuantificada de 8 bits. Entre los materiales anexados a este documento se encuentra una propuesta de programa en el fichero GenerateSinTable.m. Por otro lado el estudiante puede obtener estos valores utilizando otras herramientas como las hojas de cálculo.

\subsubsection{Configuración de la velocidad de transmisión}

Después de haber resuelto el problema de generar la sinusoide portadora el estudiante se encuentra con nuevos desafíos antes de completar el proceso de modulación. Se había mencionado que la velocidad de transmisión es de 2 kbps o que es lo mismo decir 2 kbd porque la señal de datos se modula con dos niveles. Ahora hay que determinar cuál es el mejor modo del microcontrolador 8051 para esta velocidad de transmisión. El modo 1 asíncrono UART de 8 bits con el Baud Rate variable permite asignar la velocidad de transmisión deseada. Mediante la ec. (5) se determina el valor que hay que cargarle el timer 1 en el registro $T H 1$ para obtener la velocidad de transmisión deseada.

$$
V_{t}=\frac{2^{S M O D} * \text { FrecuenciaOscilador }}{32 * 12 *(256-\text { TH } 1)}
$$

Conociendo que la frecuencia del oscilador es de $12 \mathrm{MHz}$, $S M O D=0$ y la $V_{t}=2 \mathrm{kbps}$, se realiza un despeje de TH1 y se obtiene un resultado de 241. Aunque este resultado no es exacto, para ese valor de TH1 es equivalente a una velocidad de transmisión de 2,08 kbps resultado que no afecta al sistema.

La ec. (5) se recibe como parte del contenido de transmisión del puerto serie de la asignatura Microprocesadores II y forma parte de uno de los contenidos que se evalúa en la etapa. La misma se encuentra en las hojas del fabricante del 8051. Es un logro poder motivar la búsqueda de información en las hojas de los fabricantes de diversos circuitos integrados y esta expresión es una buena oportunidad para ello. Es muy ventajoso la utilización por parte de los estudiantes de esta documentación ya que propicia un contexto de diseño más cercano al del ejercicio de la profesión.

\subsubsection{Modulación}

Al término de este análisis, los estudiantes comienzan a utilizar herramientas software para el diseño y prueba del módem. La plataforma Proteus Design Suite es un software de automatización de diseño electrónico, desarrollado por Labcenter Electronics Ltd., que ha utilizado el estudiante durante la carrera debido especialmente a las asignaturas de Electrónica Digital y Electrónica Analógica. Cabe señalar que durante todo el diseño los estudiantes tendrán momentos de prueba y error. Es decir, el diseño final se

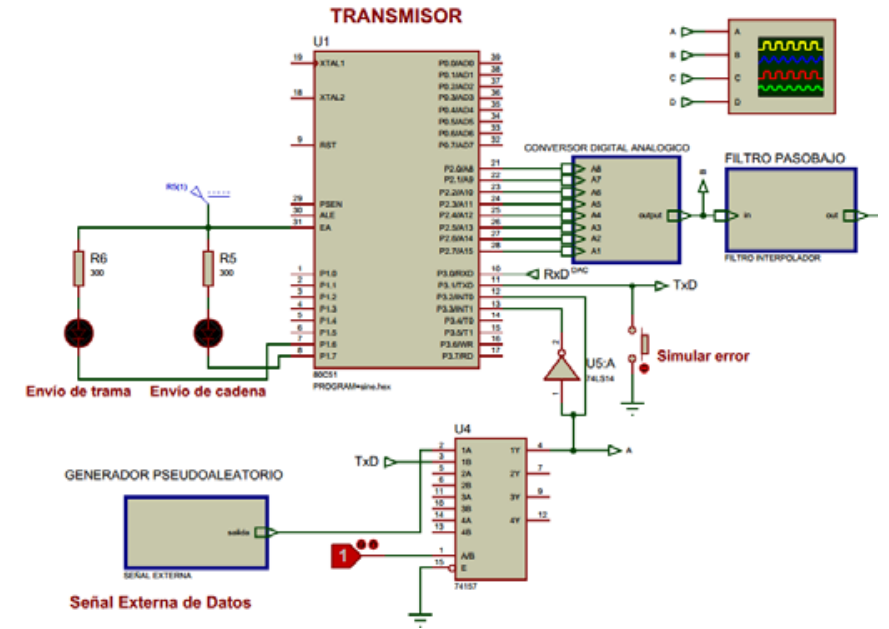

Figura 4. Esquema eléctrico del transmisor Fuente: Los autores

construye en etapas de cálculo y simulación. Este es uno de los momentos en que se evidencia el fortalecimiento de competencias relacionadas con habilidades de llevar un diseño calculado hacia un esquema eléctrico y finalmente a un programa.

Después de haber configurado la velocidad de transmisión del puerto serie del 8051 se procede a explicar cómo modular los bytes a transmitir. Una posible variante que se propone es mediante la conexión del pin TxD del 8051 a sus interrupciones externas mediante un inversor Schmitt Triggered 74LS14. Este hardware externo implica que va a ocurrir una atención a interrupción cada vez que haya un flanco de caída y un flanco de subida. Esta técnica persigue que cuando haya un flanco de subida se comienza a generar la portadora por el puerto P2 y cuando sea un flanco de caída se detiene la generación de la portadora, sacando $0 \mathrm{~V}$ a la salida del conversor digital analógico DAC. Esta conexión se puede apreciar en el esquema eléctrico del transmisor de la Fig. 4.

También se observa en el bloque del transmisor el multiplexor 74LS157 que se utiliza para seleccionar la señal digital que se quiere modular que puede estar tanto en la memoria interna del 8051 como ser una señal externa que llega al módem. Este circuito digital es opcional.

El conversor digital analógico que se utiliza es el DAC0808 en la configuración típica de aplicación que se encuentra en el datasheet a diferencia que tiene un potencial de $-5 \mathrm{~V}$ en el pin VREF para asegurar que la sinusoide tome valores entre $-5 \mathrm{~V}$ y $5 \mathrm{~V}$. Esta configuración se puede ver en detalles en el fichero de simulación de Proteus dentro del bloque con nombre DAC que se aprecia en la Fig. 4. En este punto es importante que el profesor considere explicarle al estudiante la relevancia de las demoras que introducen los circuitos integrados que utiliza ya que al tratarse de una aplicación de comunicación y pueden influir en la velocidad de transmisión.

La señal a la salida del DAC se encuentra cuantificada razón por la que hay que filtrarla con un filtro pasa bajos para suavizar estos cambios. En la asignatura de Fundamentos de las Comunicaciones II se estudia el filtro interpolador que es el que se utiliza para recuperar una señal después de haber sido muestreada siempre que se cumpla el teorema de Nyquist. El 
estudiante ha recibido contenidos sobre el diseño de filtros activos en la asignatura de Circuitos Eléctricos III durante el tercer año de la carrera. Por esta razón el diseño de este filtro con una frecuencia de corte de $2 \mathrm{kHz}$ constituye una práctica relevante en este ejercicio ya permite la ejercitación de un habilidad en un contexto de aplicación de comunicación. En la Fig. 4 se encuentra una propuesta en el bloque FILTRO PASOBAJO que se puede consultar con mayor detalle el fichero de Proteus anexo al documento.

\subsection{Implementación del receptor}

Después de la implementación del transmisor se procedería a desarrollar la etapa demoduladora del receptor. Durante la realización de esta etapa el estudiante se enfrenta al reto de cómo demodular la señal de ASK. Este se considera uno los desafíos técnicos más complejos de este ejercicio por lo que se invita al profesor a acompañar y guiar esta etapa de diseño en el espacio de aprendizaje. En esta propuesta, los bloques del demodulador fueron implementados utilizando hardware externo al 8051. En la Fig. 5 se puede apreciar esta propuesta que es continuación de la Fig. 4.

Una forma de lograr la implementación consiste en interpretar las etapas de la señal que muestra el osciloscopio en la Fig. 3. Se aconseja especialmente el trabajo de interpretación sobre estas señales con la ayuda del profesor. Al analizar la señal d) se nota una característica significante. El demodulador balanceado, que es la multiplicación de la señal ASK por una sinusoide a la misma frecuencia que la portadora y sincronizada, genera un efecto de rectificación de onda completa con una amplificación en la señal a la salida. Este efecto muestra que no es necesario sincronizar la portadora entre el transmisor y el receptor ya que rectificar la señal ASK evita la implementación de la multiplicación con la portadora local en el receptor. De manera que es posible la implementación de un rectificador de onda completa utilizando un puente diodo por ejemplo. Es un contenido que se recibe en el segundo año de la carrera en la asignatura Electrónica Analógica I para realizar la conversión de AC-DC y se puede observar como también cumple una función en este tipo de aplicación.

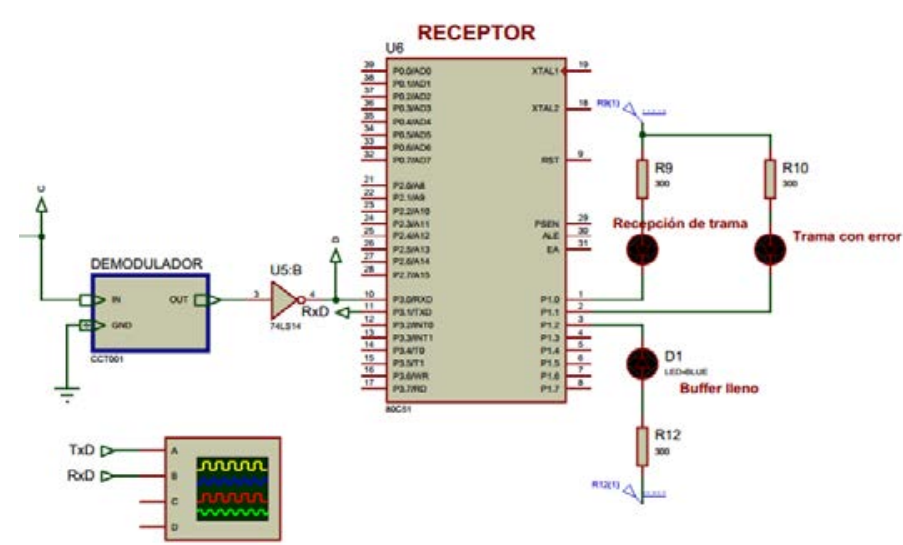

Figura 5. Esquema eléctrico del receptor Fuente: Los autores

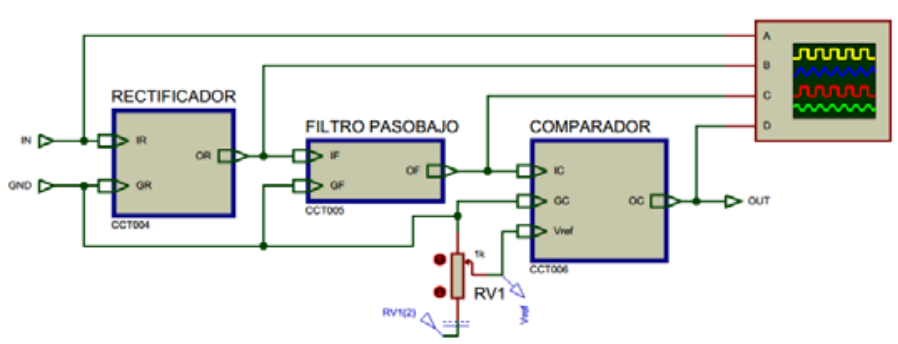

Figura 6. Bloques del demodulador

Fuente: Los autores

En esta propuesta se usa un rectificador de onda completa de precisión ya que la caída de voltaje producida por un puente diodo varía la amplitud de la señal. El rectificador de precisión de onda completa se estudia como ejemplo de aplicación de los amplificadores operaciones en la asignatura Electrónica Analógica II y el diseño propuesto se encuentra en la literatura básica Circuitos Microelectrónicos: Análisis y Diseño [10]. En la Fig. 6 se pueden observar los bloques correspondientes al demodulador.

Posteriormente al rectificador, la señal a la salida es filtrada con un filtro pasa bajo para dejar pasar las bajas componentes espectrales que van conformando la señal de datos. El filtro se diseña con los parámetros utilizados en la simulación de Simulink de la Fig. 2. A continuación un comparador de umbral define la forma de los pulsos de la señal de datos. Este comparador se construye con un amplificador operacional en régimen no lineal, donde el umbral es determinado experimentalmente mediante la simulación en Proteus con un potenciómetro. El rectificador de precisión de onda completa, el filtro pasa bajo y el comparador de umbral constituyen los bloques RECTIFICADOR, FILTRO PASOBAJO y COMPARADOR respectivamente en la Fig. 6 que forman el bloque DEMODULADOR de la Fig. 5 Para mayores detalles de estos diseños revisar los materiales anexos.

En este punto el estudiante al intentar conectar el demodulador al pin RxD del 8051 se encontrará con el problema de la incompatibilidad ya que el 8051 no interpretará correctamente los niveles digitales de la señal a la salida del bloque COMPARADOR. Esto se debe a que el amplificador operacional utilizado como comparador no brinda los niveles necesarios de tensión y corriente a la salida. Para solucionar este problema en esta propuesta se usa el inversor 74LS14 como buffer para adecuar los niveles de tensión y corriente a la salida del comparador. Este es un contenido de gran relevancia que se imparte en la asignatura de Electrónica Digital I sobre la compatibilidad entre las familias digitales y es un ejemplo de cómo se puede utilizar ese conocimiento cuando aparece la necesidad en esta experiencia específica.

En esta etapa es trascendente cómo el estudiante logra integrar los conocimientos y habilidades de las principales configuraciones de los amplificadores operacionales recibidos en la asignatura Electrónica Analógica II en un solo bloque demodulador Es importante señalar que el sentido de integración en este ejemplo no se queda solo en la unión de diversos conocimientos sino que se acerca a un nuevo conocimiento generado por esta experiencia debido a que el 
resultado de esta distribución de amplificadores operacionales ha generado el comportamiento de un demodulador. Para el mejor conocimiento de los autores no se ha encontrado hasta el momento una distribución igual en la literatura.

\subsection{Detección de errores en la transmisión}

Debido a posibles efectos indeseados sobre el canal de transmisión como el ruido y la interferencia pueden ocurrir errores en la transmisión. Es importante que el estudiante que se encuentre realizando el ejercicio conozca las causas que originan estos efectos y diversas formas de darle solución al problema. Al estar recibiendo horizontalmente la asignatura de Redes I comienza a familiarizarse con los protocolos de comunicación y diversos algoritmos de detección de errores. Es necesario entonces la implementación de uno de estos algoritmos para entender con mayor profundidad la naturaleza de los protocolos de comunicación y de esta forma se evidencia en la propuesta de ejercicio la relación con la disciplina Sistemas de Telecomunicaciones.

Para darle solución al problema se implementa el protocolo de parada y espera que se encuentra en la literatura básica de la asignatura de Redes I [11]. Consiste en que el transmisor envía una trama y no vuelve a transmitir la próxima hasta que el receptor haya enviado un byte de confirmación indicando que se recibió la trama anterior. Si hubo algún error el receptor envía un byte indicador de error para que el transmisor reenvíe la trama. Constituye un protocolo de simple implementación orientado a la conexión con confirmación de recepción. Para saber si ocurrió un error se utiliza la suma de comprobación en cada trama transmitida.

Para comprobar la implementación del protocolo, entre el transmisor y el receptor se enviará la cadena de caracteres "HolaModemLoco" que se encuentra grabada en el transmisor. Esta cadena de caracteres se enviará en tramas de 7 bytes cada una, en las cuales 6 bytes corresponden a información y el último byte es la resta que se le hace a cero con los 6 bytes anteriores, es decir, es el byte de la suma de comprobación (SC). En el receptor se realiza la operación contraria, la suma de todos los bytes de la trama más SC debe ser cero para asegurar que no hubo error durante la transmisión. En la Fig. 7 se ilustra a modo de ejemplo esta cadena caracteres en transmisiones sucesivas de sus tramas. El envío de la señal de control que indica que ocurrió un error durante la transmisión se realiza directamente del receptor al transmisor utilizando el puerto serie.

\subsection{Programación}

La programación del transmisor y el receptor del esquema de la Fig.4 y Fig. 5 respectivamente resultaría muy engorrosa y compleja si se utiliza el lenguaje ensamblador de bajo nivel. Razón por la cual se sugiere la utilización de un lenguaje de mayor nivel como lo es $\mathrm{C}$ porque facilita la elaboración y comprensión de sistemas más complejos. El estudiante ha recibido en los dos primeros años de la carrera las asignaturas Introducción a la Computación, Programación I y Programación II de manera que ha fortalecido habilidades sobre la programación en $\mathrm{C}$ y esta propuesta ayuda a consolidarlos y a enfocar estas habilidades en un contexto de una aplicación de comunicación. El ejercicio de programar un protocolo de

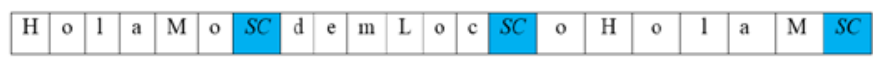

Figura 7. Tramas de la cadena de caracteres a transmitir Fuente: Los autores

comunicación y ponerlo a funcionar es una tarea que pocas veces se ha manifestado en la carrera y que pudiera ser significativa en una mejor comprensión del contenido y en la creación de nuevas habilidades. La propuesta del programa en $\mathrm{C}$ aparece en los materiales anexos donde puede ser descargada y modificada para fines docentes. Solo resta que el estudiante se familiarice con compiladores que conviertan el código $\mathrm{C}$ a un fichero .hex para poder cargarlo en el Proteus, aunque versiones modernas del Proteus incorporan un compilador.

\section{Resultados}

Después de realizar el diseño del módem el estudiante debe comprobar su funcionamiento en el Proteus. Es importante señalar que el profesor debe hacer explícito la manera en que comprueba el correcto funcionamiento del diseño y motivar al estudiante a escoger esa metodología de evaluación como guía en su aprendizaje. Para ello se proponen diferentes pruebas que se pueden realizar para el análisis de los resultados. La primera de ella es comprobar la modulación y desmodulación en ASK y que la velocidad de transmisión sea la esperada. La segunda es la comprobación de la recepción de la cadena de caracteres. La tercera y última es la comprobación del funcionamiento del protocolo parada y espera y la comprobación de que retransmite las tramas defectuosas.

\subsection{Comprobación de la modulación y desmodulación}

En la Fig. 8 se puede apreciar la captura del osciloscopio de la Fig. 4 para comprobar la modulación y en la Fig. 9 la captura del osciloscopio de la Fig. 6 para comprobar la desmodulación. Como he de esperarse se puede verificar que la velocidad de transmisión es de 2 kbps y la modulación y desmodulación de

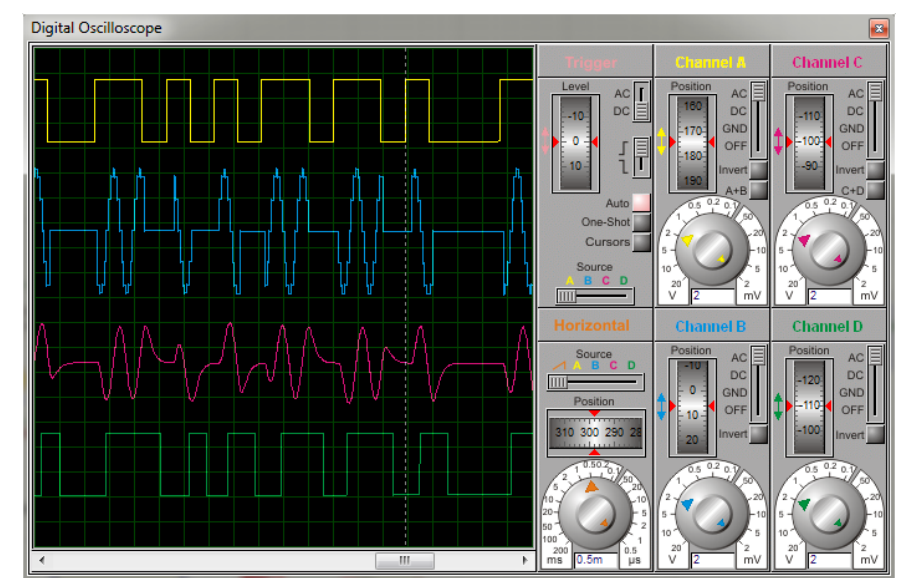

Figura 8. Captura de osciloscopio

Fuente: Los autores 


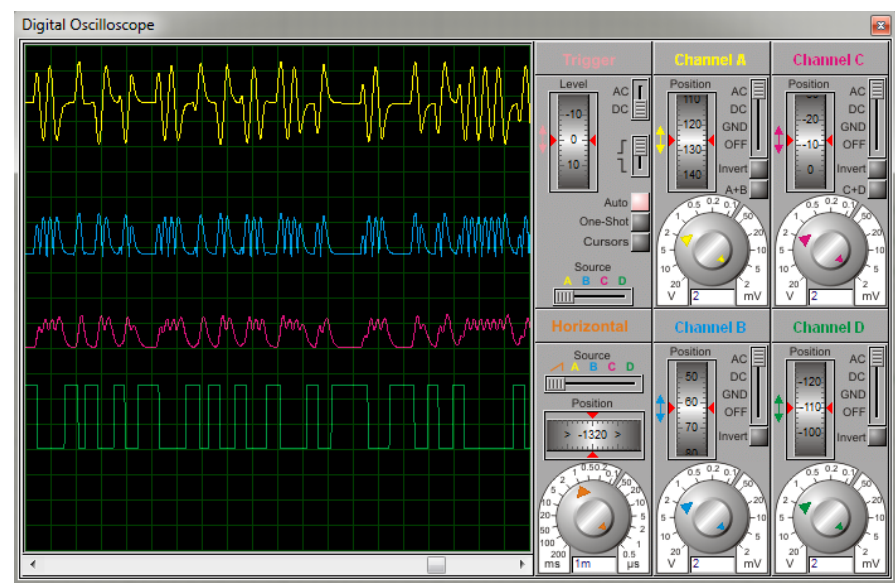

Figura 9. Captura de osciloscopio

Fuente: Los autores

8051 CPU Internal (IDATA) Memory - U6
\begin{tabular}{|l|llll|llll|l|}
\hline 00 & 00 & 00 & 00 & 00 & 00 & 00 & 00 & 00 & $\ldots . . .$. \\
08 & 48 & $6 \mathrm{~F}$ & $6 \mathrm{C}$ & 61 & $4 \mathrm{D}$ & $6 \mathrm{~F}$ & 64 & 65 & HoiaMode \\
10 & $6 \mathrm{D}$ & $4 \mathrm{C}$ & $6 \mathrm{~F}$ & 63 & $6 \mathrm{~F}$ & 48 & $6 \mathrm{~F}$ & $6 \mathrm{C}$ & mLoCoHo1 \\
18 & 61 & $4 \mathrm{D}$ & $6 \mathrm{~F}$ & 64 & 65 & $6 \mathrm{D}$ & $4 \mathrm{C}$ & $6 \mathrm{~F}$ & aModemLo \\
20 & 63 & $6 \mathrm{~F}$ & 00 & 00 & 00 & 00 & $1 \mathrm{~A}$ & 02 & Co...... \\
28 & $\mathrm{D} 2$ & 00 & 00 & 00 & 00 & 00 & 00 & 00 & $\ldots . . . .$. \\
\hline
\end{tabular}

Figura 10: Comprobación de recepción de los caracteres Fuente: Los autores

ASK se realiza correctamente. Además la frecuencia de la portadora es de aproximadamente $2 \mathrm{kHz}$ donde se puede ver cómo por cada bit de transmisión se aprecia un período de la portadora. También se aprecia la similitud de estas señales con las obtenidas en la simulación en Simulink de la Fig. 3.

\subsection{Comprobación de la transmisión de los caracteres}

Para comprobar que se realizó correctamente la transmisión de la cadena de caracteres se utiliza una opción que brinda el Proteus que permite acceder a la memoria interna del microcontrolador. Se puede acceder en el momento en que se pausa la simulación. En la Fig. 10 se aprecia cómo se almacenan los caracteres que llegan al receptor.

Para verificar el proceso de transmisión durante la ejecución de la simulación se incorporaron unos leds en el transmisor y en el receptor que se pueden observar en la Fig. 4 y Fig. 5. En el transmisor los leds cambian de estado para indicar cada vez que se envía una trama y la cadena de caracteres. En el receptor los leds indican cada vez que se recibe una trama con éxito, cuando una trama se recibió con error y cuando se llena el buffer circular para comenzar a reescribir la información.

\subsection{Comprobación del protocolo parada y espera}

Para la comprobación del protocolo parada y espera se puede verificar el proceso de transmisión de tramas y la señal de control que envía el receptor por cada trama que recibe. Esto se puede comprobar en el osciloscopio de la Fig. 5 donde se ilustra en la Fig.11. También se observa cuando se detecta un error y se envía la indicación de error al transmisor.

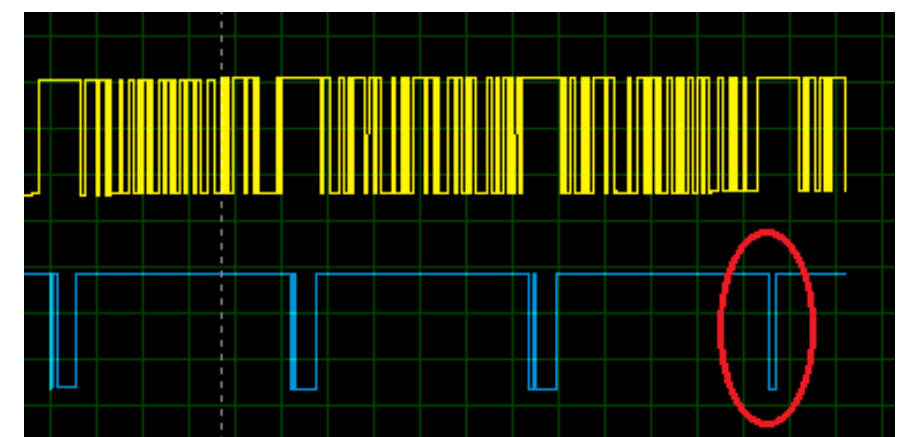

Figura 11: Comprobación del protocolo de comunicación. Pulso señalado en rojo indicador de que ocurrió un error en la suma de comprobación.

Fuente: Los autores
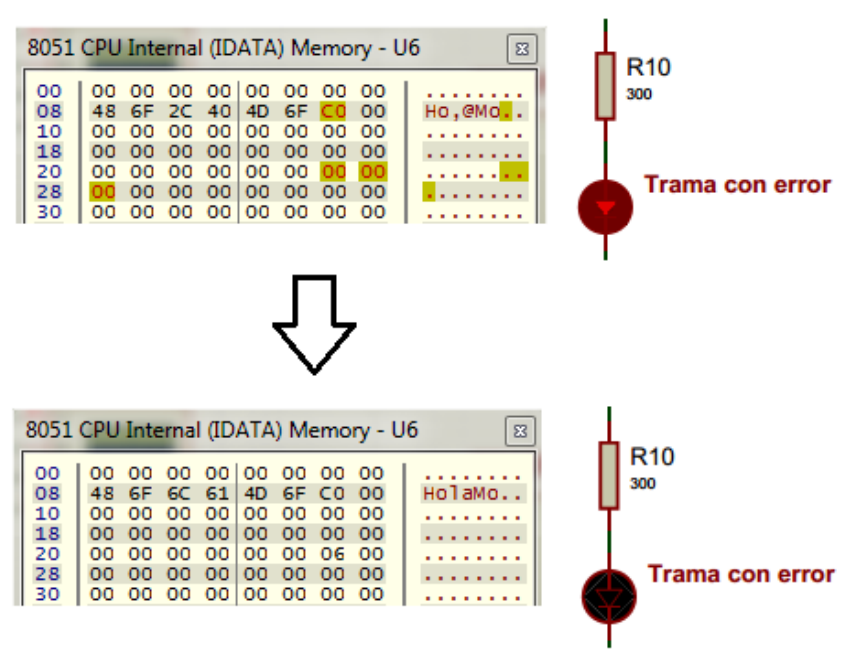

Figura 12: Comprobación del algoritmo detector de errores Fuente: Los autores

Para la comprobación de corrección del error se incorporó a la simulación un botón conectado entre el pin TxD del transmisor y tierra para que el usuario introduzca un cero lógico no deseado durante la transmisión y poder comprobar el funcionamiento del protocolo cuando detecta errores.

Se puede observar en la memoria interna del receptor en la Fig. 12 la recepción de un carácter erróneo y cómo enciende el led rojo indicando que llegó una trama defectuosa. Posteriormente la trama es retransmitida de manera que se sobrescribe el carácter erróneo con el correcto y se apaga el led rojo.

\section{Conclusiones}

En este trabajo se presentó una propuesta de un ejercicio integrador usando el microcontrolador 8051 en el diseño de un módem para la carrera de ingeniería en Telecomunicaciones y Electrónica. Se logró explicar los pasos metodológicos y técnicos para la resolución del ejercicio señalando las principales competencias que los estudiantes desarrollan en cada etapa. También se pudo exponer una relación explícita de aspectos técnicos del ejercicio con elementos del proceso de enseñanza y aprendizaje en donde el estudiante en más de una ocasión debe generar nuevos contenidos construidos con la base de conceptos y habilidades básicas ya aprendidas de otras 
disciplinas. Por lo que se puede plantear que en cierta medida esta experiencia integradora contribuye a la generación de nuevos conocimientos fundados en esta experiencia.

La forma en que los conocimientos, habilidades y aptitudes de las principales disciplinas de la carrera se conjugaron y se fortalecieron para resolver el ejercicio mostraron el carácter integrador e interdisciplinario de la propuesta.

Otra característica relevante de la propuesta es su nivel de complejidad y la importancia del trabajo en equipo de estudiantes para su resolución. La manera en que se concibió las diferentes etapas de desarrollo pudiera favorecer a la formación y fortalecimiento de valores en los equipos de estudiantes siempre que el profesor acompañe el espacio de aprendizaje.

Desde una mirada técnica, para trabajos futuros se pueden derivar otras variantes como incluir otra modulación, otro protocolo, evaluar con ruido presente en la transmisión e implementar el dispositivo en un laboratorio de electrónica. Por la parte pedagógica sería trabajar en la experimentación con varios estudiantes esta propuesta o similares para evaluar científicamente su incidencia en la formación de los mismos. Se espera y desea que esta propuesta pueda motivar el surgimiento de proyectos similares que contribuyan favorablemente al proceso de enseñanza de la carrera de Telecomunicaciones y Electrónica.

\section{Referencias}

[1] Delors, J., La educación o la utopía necesaria, Informe a la UNESCO de la Comisión Internacional sobre la Educación. La educación encierra un tesoro, pp. 13-36, 1996.

[2] Escobar, Y.C., Interdisciplinariedad: Desafío para la educación superior y la investigación, Rev Luna Azul, [En línea]. 31, pp. 156-69, 2010. Disponible en: http://www.redalyc.org/articulo.oa?id=321727233012

[3] Giraldo, A.V., et al., La interdisciplinariedad en ingeniería, [En línea]. Grupo Ingeniería y Sociedad. Facultad de Ingeniería. Universidad de Antioquia. Disponible en: http://ingenieria.udea.edu.co/producciones/ingenieria_sociedad/interdis ciplinariedad_ingenieria.pdf

[4] Barrows, H.S., A taxonomy of problem based learning methods, Medical education, 20, pp. 481-486, 1986.

[5] Gómez, B.R., Aprendizaje basado en problemas (ABP): Una innovación didáctica para la enseñanza universitaria, Educación y Educadores, [En línea]. $\quad 8, \quad 2005.2$ Disponible http://www.redalyc.org/pdf/834/83400803.pdf

[6] Betancourt-Correa, C., Aprendizaje basado en problemas una experiencia novedosa en la enseñanza de la ingeniería, Revista Educación en Ingeniería, 1(2), pp. 45-51, 2006.

[7] Fernández. F and Duarte, J., El aprendizaje basado en problemas como estrategia para el desarrollo de competencias específicas en estudiantes de ingeniería, Formación Universitaria, [En línea]. 6, pp. 29-38, 2013. Disponible

http://www.scielo.cl/scielo.php?script=sci_arttext\&pid=S071850062013000500005 DOI: 10.4067/S0718-50062013000500005

[8] Xiong, F., Digital modulation techniques: Artech House, 2006.

[9] Nyquist, H., Certain topics in telegraph transmission theory, Proceedings of the IEEE, 90, pp. 280-305, 2002.

[10] Rashid, M.H., Circuitos microelectrónicos: Análisis y diseño, International Thomson, 2000.

[11] Tanenbaum, A.S. et al., Redes de computadoras, Pearson Educación, 2012.
D. Castro-Piñol, recibió el título de Ing. en Telecomunicaciones y Electrónica en 2015 en la Universidad de Oriente de Cuba. Investiga en el campo de procesamiento de digital señales, imágenes y visión computacional. Se encuentra actualmente trabajando en el Centro de Estudios de Neurociencias, Procesamiento de Imágenes y Señales (CENPIS) de la Facultad de Ingeniería eléctrica de dicha universidad.

ORCID: 0000-0003-2906-6532

B.E. Alvarez-Portuondo, recibió el título de Ing. en Telecomunicaciones y Electrónica en 2015 en la Universidad de Oriente de Cuba. Se encuentra trabajando en el Departamento de Ingeniería Biomédica de dicha universidad donde imparte clases de electrónica analógica. Su actual interés de investigación radica en aplicaciones basadas en microcontroladores.

ORCID: 0000-0002-5170-3158

H. Hervella-Mateos, obtuvo el título de Ing. en Telecomunicaciones y Electrónica en el 2015 en la Universidad de Oriente, Cuba. Se encuentra trabajando en la Empresa de Telecomunicaciones de Cuba ETECSA. Es especialista "C" en Telemática y se encarga de gestionar la infraestructura de transmisión y datos de la capa de transporte del territorio. Su actual interés de investigación radica en las redes telemáticas.

ORCID: 0000-0001-6618-2378

I. Rivero-Pouymiró, obtuvo el título de Ing. en Telecomunicaciones y Electrónica en el 2014 en la Universidad de Oriente, Cuba. En el 2017 obtuvo el título de MSc. en Ingeniería Biomédica en la Universidad de Oriente, Cuba. Desde el 2014 es profesora de cursos de pregrado de electrónica digital y microprocesadores. Su interés de investigación está en el procesamiento digital de señales biomédicas.

ORCID: 0000-0002-6406-3794 